15

\title{
Влияние адгезии на трение качения и скольжения: эксперимент
}

\author{
() Я.А. Ляшенко, ${ }^{1,2}$ В.Л. Попов ${ }^{1,3,4}$ \\ ${ }^{1}$ Берлинский технический университет, \\ 10623 Берлин, Германия \\ ${ }^{2}$ Сумский государственный университет, \\ 40007 Сумы, Украина \\ ${ }^{3}$ Национальный исследовательский Томский государственный университет, \\ 634050 Томск, Россия \\ ${ }^{4}$ Институт фризики прочности и материаловедения, \\ 634055 Томск, Россия \\ e-mail: i.liashenko@tu-berlin.de,v.popov@tu-berlin.de \\ Поступило в Редакцию 10 июля 2021 г. \\ В окончательной редакции 20 августа 2021 г. \\ Принято к публикации 21 августа 2021 г.
}

Исследованы нормальный и тангенциальный контакты между цилиндрическим стальным индентором (колесом) и эластомером, обладающим высокими адгезионными свойствами. В случае индентирования в нормальном направлении проведено компьютерное моделирование процесса индентирования и отрыва, показавшее совпадение с экспериментом. Для режима трения качения при анализе измеренных зависимостей тангенциальной компоненты контактной силы от смещения колеса определена адгезионная составляющая силы трения. Рассмотрена ситуация трения скольжения, в которой вращение колеса заблокировано. При наличии адгезии сила трения скольжения пропорциональна площади контакта. В случае отсутствия адгезии (эластомер полностью покрыт пылью из мела) реализуется прерывистый stick-slip-режим трения. Частота и амплитуда stick-slip-переходов зависят от глубины погружения индентора в эластомер.

Ключевые слова: нормальный и тангенциальный контакты, индентирование, трение, адгезия, качение, скольжение.

DOI: $10.21883 / J T F .2022 .01 .51867 .212-21$

\section{Введение}

Адгезия играет большую роль как в живой природе, так и в разнообразных технических приложениях [1-5]. Размеры биологических организмов, активно использующих адгезионные механизмы для фиксации и перемещения, варьируются в широких диапазонах - это могут быть как бактерии $[2,3]$, так земноводные и пресмыкающиеся $[4,5]$. В качестве примера технологических процессов, в которых адгезия играет определяющую роль, можно привести склеивание поверхностей, пайку, сварку, нанесение покрытий (в частном случае покраску), фасовку удобрений и многое другое [1]. При наличии ярко выраженного адгезионного взаимодействия между контактирующими поверхностями адгезионные процессы критическим образом влияют на контактное поведение, а иногда определяют его полностью [6]. Первой строгой теорией, описывающей адгезионный контакт, является теория К.Л. Джонсона, К. Кендала и А.Д. Робертса (JKR-теория) [7,8]. Подход JKR исходит из предположения, что расстояния, на которых действуют адгезионные силы, ничтожно малы по сравнению с геометрическими параметрами контакта - такими как глубина индентирования и радиус контакта. Следующей значимой теорией, которая описывает адгезионный контакт в случае дальнодействующего адгезионного взаимодействия, является теория Б.В. Дерягина,
В.М. Мюллера и Ю.П. Топорова (DМТ-теория) [9]. Позже в работе [10] был продемонстрирован переход между этими двумя предельными случаями при изменении характерного расстояния убывания адгезионных сил. В настоящее время адгезионный контакт активно исследуется различными научными группами как экспериментально [6,11-13], так и теоретически [14-16]. Экспериментальные работы по изучению прочности адгезионного контакта на отрыв показывают, что в системах, имеющих совершенно различные масштабы, существуют общие закономерности $[6,12]$. Например, в процессе индентирования адгезионный контакт распространяется гораздо хуже, чем это предсказывает классическая теория JKR, в то время как при разрушении контакта он характеризуется значительно большей адгезионной прочностью. Это приводит к гистерезису зависимостей „сила-перемещение“ при изменении направления движения индентора, что отсутствует в классических подходах. Наличие гистерезиса при нагружении и дальнейшей разгрузке контакта объясняется действием различных механизмов, связанных с вязко-упругостью, влажностью и пластичностью [17]. Однако достоверные причины, приводящие к гистерезису, до сих пор до конца не выяснены. Обсуждаемый гистерезис является причиной дополнительной диссипации механической энергии в осциллирующем адгезионном контакте [18]. Также он 
приводит к различному поведению границы контакта на переднем и заднем фронте при тангенциальном сдвиге [6]. В частности, в проводимых нами экспериментах неоднократно наблюдалось, что возникновение новых областей контакта происходит скачкообразно, в то время как при разрушении контакта его граница является более гладкой $[6,18,19]$. Эта особенность справедлива не только для нормального контакта, но и для тангенциального сдвига, в котором на переднем фронте возникает новый контакт, а на заднем фронте адгезионный контакт разрушается.

В работе [6] описаны особенности тангенциального сдвига (трения) между эластомером и стальным сферическим индентором при различных радиусах индентора и глубинах индентирования. В указанной работе было показано, что во всех рассмотренных случаях реализуется режим, в котором внутри зоны контакта устанавливается постоянное значение сдвиговых напряжений, которые в основном и задают силу трения. Однако сила трения имеет еще одну компоненту, связанную с диссипацией механической энергии на границе контакта. Составляющая силы трения, связанная с постоянными сдвиговыми напряжениями, зависит от их величины и площади контакта, поэтому может быть определена из эксперимента, в то время как трение на границе контакта - это более тонкий процесс, в ходе которого на протяжении тангенциального сдвига производится постоянная перестройка геометрии границы контакта, наблюдаемая в эксперименте визуально [6]. В настоящей работе мы описываем результаты экспериментов по изучению трения качения колеса, вдавленного в эластомер на различную глубину. Целью проведенных экспериментов являлось установление влияния адгезионного взаимодействия между контактирующими телами на трение. Отметим, что в [6] было проведено компьютерное моделирование трения качения в рамках метода граничных элементов. В настоящей работе мы показали, что в реальности процесс трения качения гораздо сложнее модельных представлений, что, в частности, вызвано внутренними процессами, происходящими в подшипнике качения. Все описываемые ниже эксперименты были проведены при малых скоростях движения индентора - от 1 до $5 \mu \mathrm{m} / \mathrm{s}$. Настолько малые скорости выбирались для того, чтобы в каждый момент времени контакт можно было считать квазистатическим. При этом становится возможным проведение сравнения результатов экспериментов с классическими теориями и результатами компьютерного моделирования.

\section{1. Экспериментальная установка для исследования нормального и тангенциального контактов}

На рис. 1 приведена фотография разработанного устройства, с помощью которого были проведены эксперименты, описываемые далее. Устройство состоит из следующих элементов. Индентор 4 прикреплен к трехосевому сенсору силы ME K3D40 (позиция 3). Сенсор сдвигается в нормальном и тангенциальном направлениях при помощи высокоточных приводов направленного движения M-403.2DG (позиции 1 и 2), которые управляются контроллерами С-863. Согласно табличным характеристикам, данные приводы при сдвиге в одном направлении обеспечивают точность позиционирования до $1 \mu \mathrm{m}$, но при изменении направления движения существует люфт величиной до $10 \mu \mathrm{m}$, что нужно учитывать при обработке экспериментальных результатов. Для усиления электрического сигнала, получаемого от сенсора силы, используется четырехканальный аналоговый усилитель GSV-1A4 SubD37/2. Сопряжение усилителя с компьютером осуществляется с помощью 16-битного АЦП NI USB-6211. В эксперименте происходит вдавливание индентора 4 в эластомер 5 , в роли которого выступает лист прозрачной резины TARNAC CRG N3005 с толщиной $5 \mathrm{~mm}$. Резиновый лист находится на пластине из силикатного стекла, к которой прочно прикрепляется за счет собственной адгезии, без использования дополнительных крепежей либо клея. Наблюдение за эволюцией контактной области осуществляется снизу контакта с помощью цифровой видеокамеры Conrad USB с физическим разрешением $1600 \times 1200$ пикселей. Камера (позиция 8) скрыта от наблюдателя пластиной фиксирующего устройства. Согласованное управление всеми электронными устройствами осуществляется с помощью компьютерной программы, разработанной в среде программирования LabVIEW. Сценарий движения индентора задается в виде двух отдельных функций времени, согласно которым происходит движение приводов 1 и 2. На протяжении эксперимента с временным интервалом $1 \mathrm{~s}$ записываются значения трех компонент контактной силы и сохраняются фотографии области контакта. Поскольку движение осуществляется с очень маленькой скоростью (от 1 до $5 \mu \mathrm{m} / \mathrm{s}$ ), интервал сохранения показаний приборов длительностью $1 \mathrm{~s}$ позволяет подробно проследить эволюцию контактных сил и формы контакта. При необходимости вычисляется площадь контакта с помощью компьютерного алгоритма при анализе фотографий контактной области. Данный алгоритм основан на различии цвета пикселей на цифровых снимках, которые соответствуют наличию либо отсутствию контакта, поэтому важно, чтобы область контакта индентора с эластомером была равномерно освещена, что обеспечивается всесторонним светодиодным освещением 6. Номером 7 на рисунке показан наклонный механизм, позволяющий изменять положение в пространстве образца, в который происходит индентирование. В экспериментах с наличием тангенциального движения это особенно важно, поскольку наклонный механизм позволяет обеспечить параллельность поверхности эластомера и траектории движения индентора. Данная установка использовалась нами ранее для получения экспериментальных результатов, описанных в некоторых предыдущих работах [6,18-20]. 

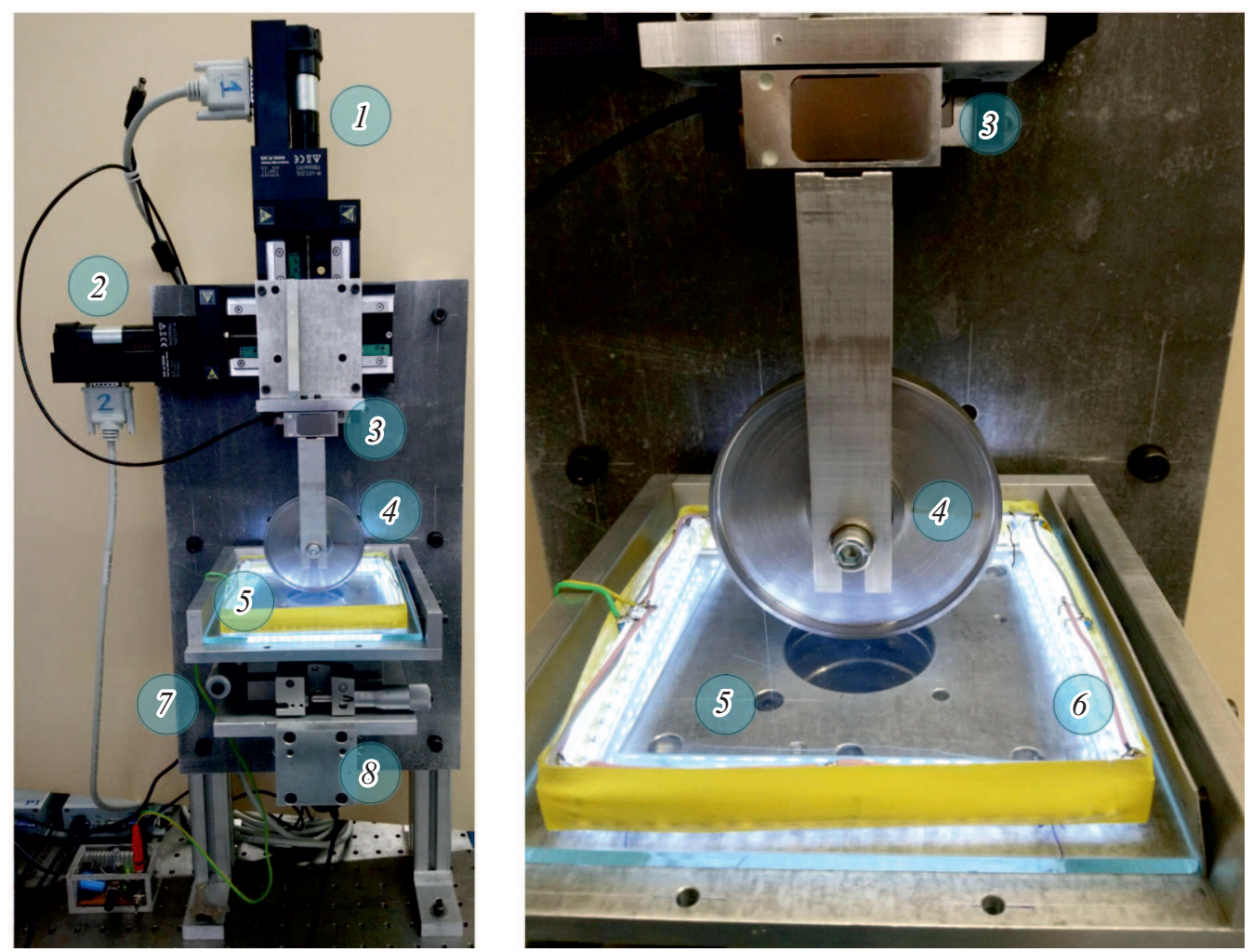

Рис. 1. Левая панель - внешний вид экспериментальной установки; правая панель - крупный план области контакта со всесторонним светодиодным освещением.

\section{2. Индентирование в нормальном направлении}

Перед тем, как перейти к исследованию тангенциального движения индентора, была проведена серия экспериментов по нормальному индентированию на глубину $d=0.3 \mathrm{~mm}$. Скорость движения индентора в этой серии экспериментов составляла $v=1 \mu \mathrm{m} / \mathrm{s}$. Индентирование производилось в лист оптически прозрачной резины TARNAC CRG N3005 с толщиной $h=5 \mathrm{~mm}$ и размерами $100 \times 100 \mathrm{~mm}$, которая находилась на пластине из силикатного стекла (рис. 1). После окончания фазы индентирования осуществлялся отрыв индентора до полного разрушения контакта. Полученные результаты показаны на рис. 2. На рис. 2, $a$ и $b$ проиллюстрированы зависимости нормальной силы $F_{N}$ и площади контакта $A$ от глубины индентирования $d$. Сплошными линиями показаны результаты трех подряд проведенных экспериментов, где кривые $A B C$ отвечают фазе индентирования, а путь $C D E F-$ фазе отрыва. Все три эксперимента показывают хорошую воспроизводимость результатов, поскольку кривые накладываются друг на друга. На рис. 2, с приведены фотографии области контакта, соответствующие точкам, обозначенным на панелях $a$ и $b$ рисунка. При этом следует отметить, что форма контакта отклоняется от прямоугольной. Это вызвано как неизбежным присутствием геометрических и химических неоднородностей на поверхностях резины и индентора, так и более высокой концентрацией напряжений в угловых точках области контакта.

Символами на рис. 2, $a$ и $b$ показаны зависимости, полученные в результате компьютерного моделирования процесса индентирования методом граничных элементов с учетом конечной толщины резиновой подложки. Подробно используемый метод численного моделирования описан в работе [21]. В моделировании использовались следующие параметры резиновой подложки: модуль упругости $E=0.35 \mathrm{MPa,} \mathrm{коэффициент} \mathrm{Пуассо-}$ на $v=0.47$, толщина листа резины $h=5 \mathrm{~mm}$. Удельная работа адгезии на стадии вдавливания индентора $\gamma_{12}=0.0175 \mathrm{~J} / \mathrm{m}^{2}$, на стадии отрыва $-\gamma_{12}=0.5 \mathrm{~J} / \mathrm{m}^{2}$. Геометрические параметры индентора: радиус колеса $R=40 \mathrm{~mm}$, его толщина $H=11 \mathrm{~mm}$. Используемые в моделировании толщина резины $h$, глубина индентирования $d$, а также геометрические параметры индентора $R$ и $H$ точно совпадают с экспериментом. Значения модуля упругости $E$ и удельной работы адгезии $\gamma_{12}$ немного отличаются от используемых нами ранее и выбраны такими, чтобы обеспечить максимальное согласование экспериментальных и теоретических результатов. Отличие в параметрах используемой резины TARNAC связано с тем, что они не являются строго постоянными и зависят от условий эксперимента и от конкретного листа резины, особенно сильно отличается удельная работа адгезии [20]. 

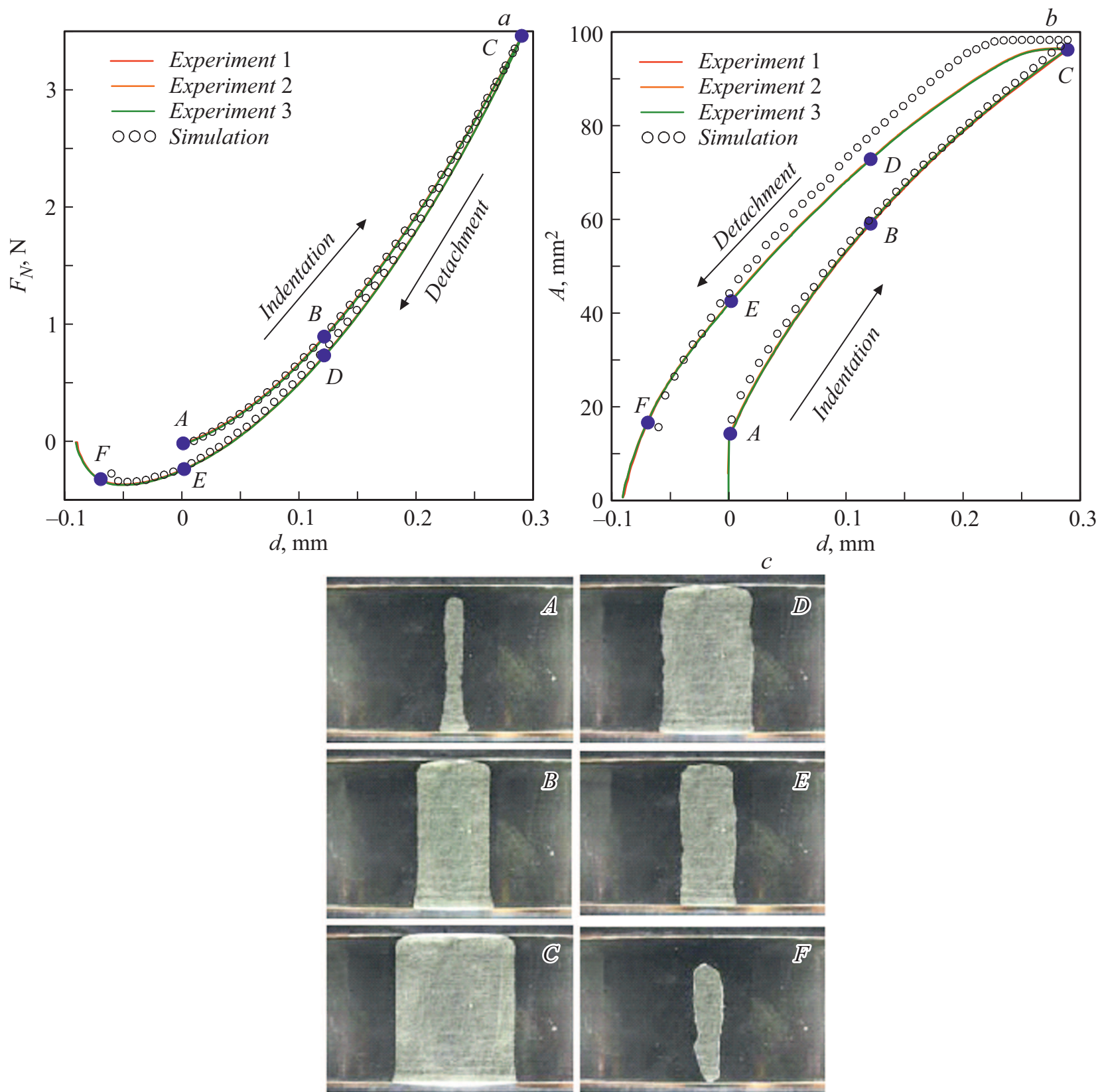

Рис. 2. Зависимости нормальной силы $F_{N}(a)$ и площади контакта $A(b)$ от глубины индентирования $d$. Сплошные линии соответствуют трем подряд проведенным экспериментам, символы показывают результаты компьютерного моделирования. На панели $c$ показаны экспериментальные фотографии контактной области, соответствующие точкам на панелях $a$ и $b$. Ход эксперимента продемонстрирован на видео (пункт 1 Приложения), которое доступно в онлайн-версии статьи.

На рис. $2, b$ и $c$ видно, что в точке возникновения первого контакта (точка $A$ ) область контакта скачкообразно распространяется за счет адгезии до величины $A \approx 15 \mathrm{~mm}^{2}$. Такому распространению соответствует довольно низкое значение удельной работы адгезии $\gamma_{12}=0.0175 \mathrm{~J} / \mathrm{m}^{2}$, найденное при сравнении эксперимента с результатами моделирования процесса индентирования (см. части зависимостей $A B C$, показанные на рисунке символами). При такой малой удельной работе адгезии упругая сила $F_{N}$ в точке первого контакта принимает близкие к нулевому отрицательные значения. В фазе отрыва реализуется гораздо бо́льшая удельная работа адгезии $\gamma_{12}=0.5 \mathrm{~J} / \mathrm{m}^{2}$. Это приводит к гистерезису зависимостей $F_{N}(d)$ и $A(d)$, который легко наблюдать на рис. 2. Таким образом, как и в предыдущих экспериментах [18-20], адгезия проявляется в основном на стадии отрыва, а предшествующей ей стадии индентирования соответствует гораздо меньшее значение удельной работы адгезии.

Следует отметить, что в эксперименте начальное распространение контакта осуществляется немного иначе, чем это показано на рис. 2. Точка первого контакта 
из-за наличия неоднородностей приходится на край индентора (рис. 2,c). Затем контакт медленно нарастает, после чего скачкообразно увеличивается до $A \approx 15 \mathrm{~mm}^{2}$. Момент резкого увеличения контакта соответствует глубине индентирования $d \approx 0.012 \mathrm{~mm}$, которая при обработке зависимостей, показанных на рис. 2, полагается нулевой, поэтому на рисунке экспериментальные зависимости продолжаются не до максимального значения $d=0.3 \mathrm{~mm}$, а до $d=0.3-0.012=0.288 \mathrm{~mm}$. Описанные манипуляции произведены для того, чтобы исключить особенности начального распространения контакта, вызванные отклонениями контактирующих тел от правильной геометрической формы. При этом становится возможным численное сравнение с результатами моделирования, в котором контактирующие поверхности имеют правильную геометрическую форму. В прилагаемом видео (см. пункт 1 Приложения) показаны необработанные результаты эксперимента, поэтому зависимости на видео отличаются от приведенных на рис. 2 в настоящей работе.

При сравнении экспериментальных результатов с результатами моделирования видно, что в эксперименте контакт начинает разрушаться раньше (рис. 2,b). Это связано с тем, что в моделировании используются геометрически правильная форма индентора и идеально гладкая поверхность эластомера, также в зоне контакта отсутствуют какие-либо неоднородности. При этом поверхности индентора и эластомера, находящиеся в контакте, параллельны друг другу. Именно в таком случае достигается максимальная прочность контакта, поэтому при изменении направления движения индентора на отрыв площадь контакта будет сохраняться максимально долго. В эксперименте контакт, как правило, начнет разрушаться раньше - на существующих химических и геометрических неоднородностях, неровностях, частицах износа и т.п. Такое поведение наблюдалось нами ранее в [19] для контакта стальной сферы с эластомером.

\section{3. Тангенциальный сдвиг - качение}

\section{1. Адгезионные свойства тангенциального контакта}

Эксперименты по исследованию тангенциального движения проводились следующим образом. Сначала индентор (колесо) погружался на фиксированную глубину $d$, после чего прокатывался в тангенциальном направлении на расстояние $x=15 \mathrm{~mm}$, а затем в обратном направлении до первоначальной координаты $x=0 \mathrm{~mm}$. После этого осуществлялся подъем индентора в нормальном направлении вверх до полного исчезновения контакта. Скорость движения во всех фазах составляла $v=5 \mu \mathrm{m} / \mathrm{s}$, что в 5 раз больше, чем в описанном выше эксперименте с нормальным индентированием. Более высокая скорость движения индентора позволяет сократить время эксперимента, но при этом она остается достаточно низкой для того, чтобы контакт можно было считать квазистатическим [20]. На рис. 3 показаны измеренные в эксперименте зависимости нормальной $F_{N}$ и тангенциальной $F_{x}$ компонент контактной силы от величины тангенциального смещения $x$ для нескольких подряд проведенных экспериментов. Все приведенные зависимости состоят из двух частей, соответствующих движению индентора (колеса) в противоположных направлениях.

Рис. 3, $a$ и $b$ соответствуют эксперименту, в котором осуществлялся прямой контакт между индентором и резиной. Целью проведенных исследований являлось выяснение влияния адгезии на трение качения. В рассматриваемой ситуации на получаемые зависимости контактных сил сильное влияние могут оказывать процессы, происходящие внутри подшипника качения (рис. 1). Для выяснения влияния этих процессов на измеряемую тангенциальную силу была проведена серия дополнительных экспериментов, результаты которых показаны на рис. 3, $c$ и $d$. В этих экспериментах на поверхность резины был нанесен тонкий равномерный слой пыли, состоящей из мела. Мел наносился с целью исключения адгезионного взаимодействия, которое имеет место при наличии прямого контакта между индентором и резиной. При сравнении „адгезионного“ (рис. 3, $a$ и $b$ ) и „безадгезионного“ (рис. 3, c и $d$ ) случаев представляется возможным оценить влияние адгезии на процесс трения качения в рассматриваемой системе. В безадгезионном случае было проведено подряд 4 эксперимента, соответствующие кривые показаны различными цветами. Из рис. $3, d$ видно, что все 4 эксперимента показывают практически идентичные зависимости $F_{x}(x)$. При этом зависимости $F_{x}(x)$ имеют выраженные осцилляции, характер которых не изменяется при изменении направления движения. Эти осцилляции не являются функцией времени, а зависят от координаты индентора и связаны с ориентацией колеса. Мы предполагаем, что наблюдаемые осцилляции связаны с внутренними процессами в подшипнике качения, поскольку они присутствуют как в адгезионном, так и в безадгезионном случаях. Рассмотрим основные различия между ситуациями наличия и отсутствия адгезионного взаимодействия. В адгезионном случае на протяжении тангенциального движения амплитуда наблюдаемых осцилляций силы $F_{x}(x)$ выше, т. е. реализуются бо́льшие значения тангенциальной силы. Подряд было проведено 6 экспериментов, результаты которых приведены на рис. 3, $a$ и $b$. Из рисунков следует, что в каждом последующем эксперименте амплитуда осцилляций тангенциальной силы уменьшается. Результаты последних двух экспериментов показаны штриховыми линиями. Зависимости $F_{x}(x)$, соответствующие штриховым линиям, находятся близко друг к другу. Таким образом, в каждом последующем эксперименте амплитуда изменения тангенциальной силы $F_{x}(x)$ уменьшается. При этом зависимость $F_{x}(x)$ стремится к некоторой стационарной форме, которая при проведении последующих экспериментов не изменяется. Проведенные исследования показывают, что эта стационарная форма $F_{x}(x)$ соответствует безадгезионному случаю. 

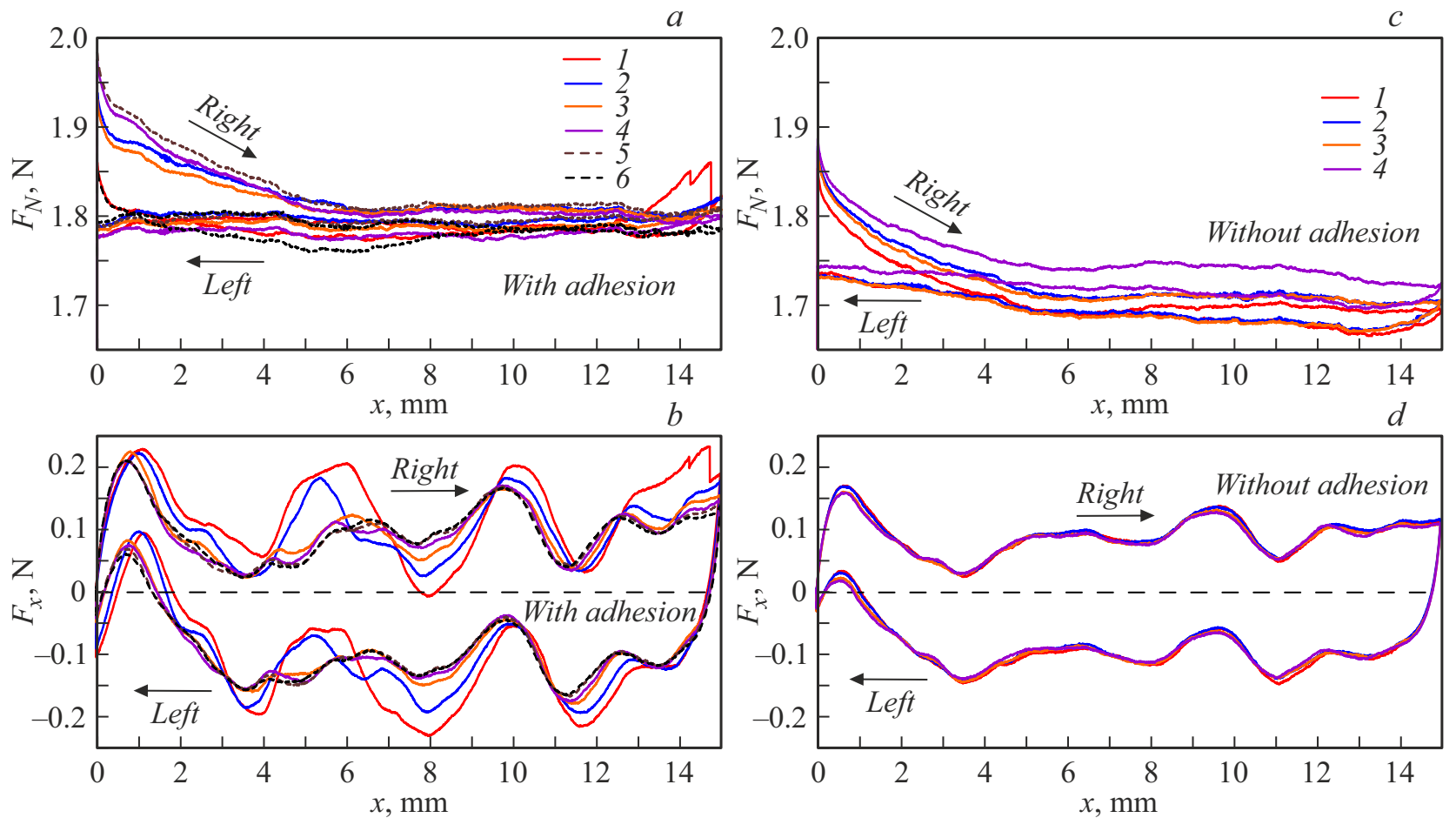

Рис. 3. Зависимости нормальной $F_{N}$ и тангенциальной $F_{x}$ контактных сил от тангенциального смещения индентора $x$; $a$ и $b$ соответствуют случаю наличия адгезии между контактирующими телами; $c$ и $d$ отвечают безадгезионному случаю. Ход эксперимента показан на видео (пункт 2 Приложения), где продемонстрированы зависимости от времени нормальной и тангенциальной сил, а также зависимость тангенциальной силы от координаты, дополненные фотографиями контактной области. На видео показаны два эксперимента, соответствующие первым циклам индентирования в адгезионном (рис. 3, $a, b$ ) и безадгезионном (рис. $3, c, d$ ) случаях. Видео доступно в онлайн-версии статьи.

Уменьшение амплитуды колебаний силы $F_{x}(x)$ связано с уменьшением удельной работы адгезии в контакте резина - сталь за счет загрязнения контактирующих поверхностей после их взаимодействия, а также за счет окисления поверхности стального индентора [20]. Ранее нами неоднократно наблюдался эффект уменьшения адгезионной прочности в экспериментах по изучению разрушения нормального контакта, а именно при многократном индентировании изначально высокое значение адгезионной прочности приближалось к некоторому существенно меньшему стационарному значению [20]. Такой эффект может быть связан только с уменьшением удельной работы адгезии, которая является функцией поверхностной энергии контактирующих поверхностей.

Для оценки вклада адгезии в трение качения было произведено алгебраическое вычитание „безадгезионной“" зависимости $F_{x}(x)$, полученной при усреднении всех кривых, приведенных на рис. $3, d$, из „адгезионных“ $F_{x}(x)$, показанных на рис. $3, b$. Зависимости полученной разницы между силами $\Delta F(x)$ показаны на рис. 4. Чтобы не загромождать рисунок, зависимости, соответствующие сдвигу индентора вправо и влево, приведены на разных панелях. Из рис. 4 следует, что вклад адгезии в тангенциальную силу уменьшается для каждого последующего цикла индентирования. При расчете зави- симостей $\Delta F$ из сил, показанных на рис. $3, b$, вычитался „безадгезионный“ вклад, показанный на рис. $3, d$. Поэтому на рис. 4 участки $\Delta F>0 \mathrm{~N}$ соответствуют ситуации, в которой адгезионное взаимодействие увеличивает тангенциальную силу. Интервалы $\Delta F<0 \mathrm{~N}$ отвечают участкам траектории индентора, на которых за счет дополнительного действия адгезии тангенциальная сила уменьшается. Изменение влияния адгезии на тангенциальную силу может быть объяснено схемой, изображенной на рис. 5 , где показано движение колеса по неровной поверхности. На рисунке показаны два положения колеса - при подъеме на неровность и при скатывании с нее. В проведенных нами экспериментах колесо движется с постоянной скоростью, поэтому при подъеме на неровность за счет ее деформирования будет возникать дополнительная тангенциальная сила $F_{t}$. Направление силы $F_{t}$ будет совпадать с направлением действия тангенциальной составляющей адгезионной силы $F_{a d}$, которая направлена против направления движения. Поэтому при подъеме колеса на возвышенность адгезионная сила увеличивает тангенциальную силу. При скатывании с неровности сила $F_{t}$ поменяет знак. Поскольку адгезионная составляющая $F_{a d}$ всегда направлена против направления движения, а оно не изменилось, при скатывании она будет стремиться уменьшить 

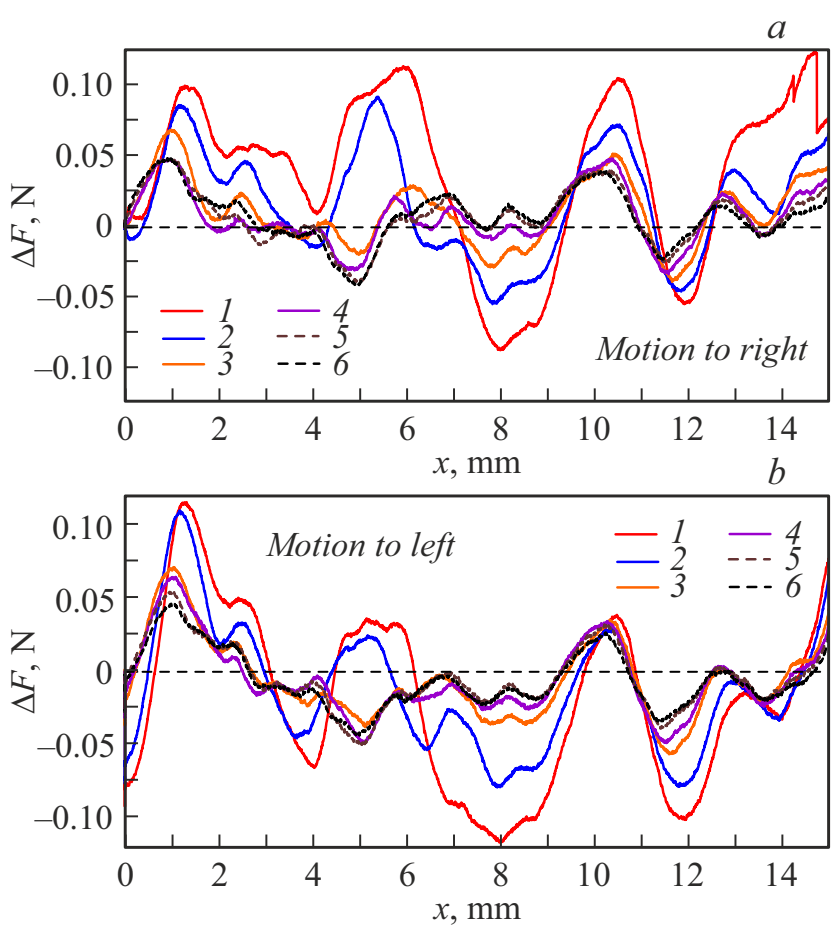

Рис. 4. Зависимости разницы между тангенциальными компонентами сил $F_{x}(x)$ в адгезионном (рис. $\left.3, b\right)$ и безадгезионном (рис. $3, d)$ случаях при движении индентора вправо (верхняя панель) и влево (нижняя панель).

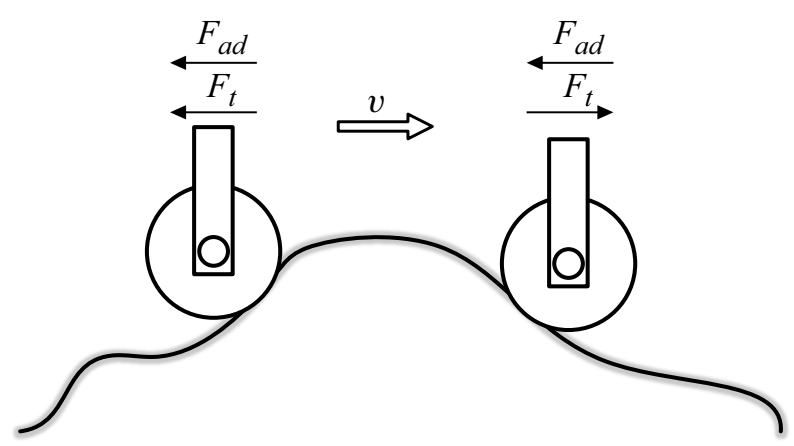

Рис. 5. Схема, поясняющая изменение направления действия тангенциальной силы за счет неровностей контактирующих поверхностей.

абсолютное значение суммарной тангенциальной силы. Эксперименты, результаты которых проиллюстрированы на рис. 3 , показали, что сила $F_{t}$ приводит к существенным колебаниям зависимости $F_{x}(x)$.

Мы предполагаем, что показанная на рис. 5 сила $F_{t}$ возникает не за счет неровностей поверхности резины, а за счет геометрических особенностей внутри подшипника качения. Это предположение основано на том, что используемая резина является визуально гладкой. Более того, если бы колебания тангенциальной силы были вызваны непосредственно неровностями резинового образца, аналогичные колебания должны были бы наблюдаться и на зависимостях нормальной силы $F_{N}(x)$, которые также показаны на рис. 3. Сила $F_{N}$ при тангенциальном движении по неровной поверхности резины должна изменяться, поскольку при движении колеса толщина резины в области контакта в случае неровной поверхности не постоянна. Однако такой связи между зависимостями $F_{x}(x)$ и $F_{N}(x)$, приведенными на рис. 3 , не наблюдается. После всех описанных в настоящей работе экспериментов была проведена дополнительная серия экспериментов для безадгезионного случая с различным начальным угловым положением колеса. Полученные результаты показали, что вид зависимости $F_{x}(x)$ существенно зависит от начального угла, на которое повернуто колесо. Это означает, что внутренние процессы, происходящие в подшипнике, влияют на получаемые результаты.

\section{2. Влияние глубины индентирования}

На рис. 6 приведены результаты экспериментов, проведенных для адгезионного и безадгезионного случаев, где каждому циклу индентирования соответствовала различная глубина индентирования $d$ (от 0.1 до $0.5 \mathrm{~mm}$ ). Из рис. 6 следует, что глубина индентирования $d$ слабо влияет на тангенциальную силу $F_{x}(x)$ как в адгезионном, так и в безадгезионном случаях, хотя нормальная сила при увеличении глубины индентирования изменяется в широких пределах - от $F_{N}<1 \mathrm{~N}$ при $d=0.1 \mathrm{~mm}$ до $F_{N}>8 \mathrm{~N}$ при $d=0.5 \mathrm{~mm}$. Если сравнить зависимости $F_{x}(x)$ в адгезионном (рис. $\left.6, b\right)$ и безадгезионном (рис. $6, d$ ) случаях, то видно, что в адгезионном случае реализуются бо́льшие абсолютные значения тангенциальной силы, т.е. адгезия приводит к дополнительной диссипации механической энергии. Однако изменения между адгезионным и безадгезионным случаями здесь не такие существенные, как, например, на рис. 3. Связано это с тем, что эксперимент, показанный на рис. 6, проводился после эксперимента, приведенного на рис. 3 . Как уже указывалось выше, каждый последующий цикл индентирования приводит к уменьшению удельной работы адгезии по разрушению контакта и соответственно к уменьшению адгезионного вклада в трение. В некоторых наших предыдущих работах по изучению адгезионной прочности перед каждым экспериментом проводилась очистка поверхностей индентора и резины, в том числе химическая. С помощью такой очистки после снижения удельной работы адгезии за счет загрязнения и окисления контактирующих поверхностей ее можно вернуть к прежнему уровню. В частности, это позволило провести серию экспериментов по изучению влияния шероховатости на адгезионную прочность [19]. В рассматриваемом в настоящей работе случае большую роль играет начальное (угловое) положение индентора (колеса). В связи с этим все описанные в работе эксперименты проводились при одном и том же начальном положении индентора, а индентор после каждого цикла индентирования не очищался. Поэтому каждый последующий эксперимент 

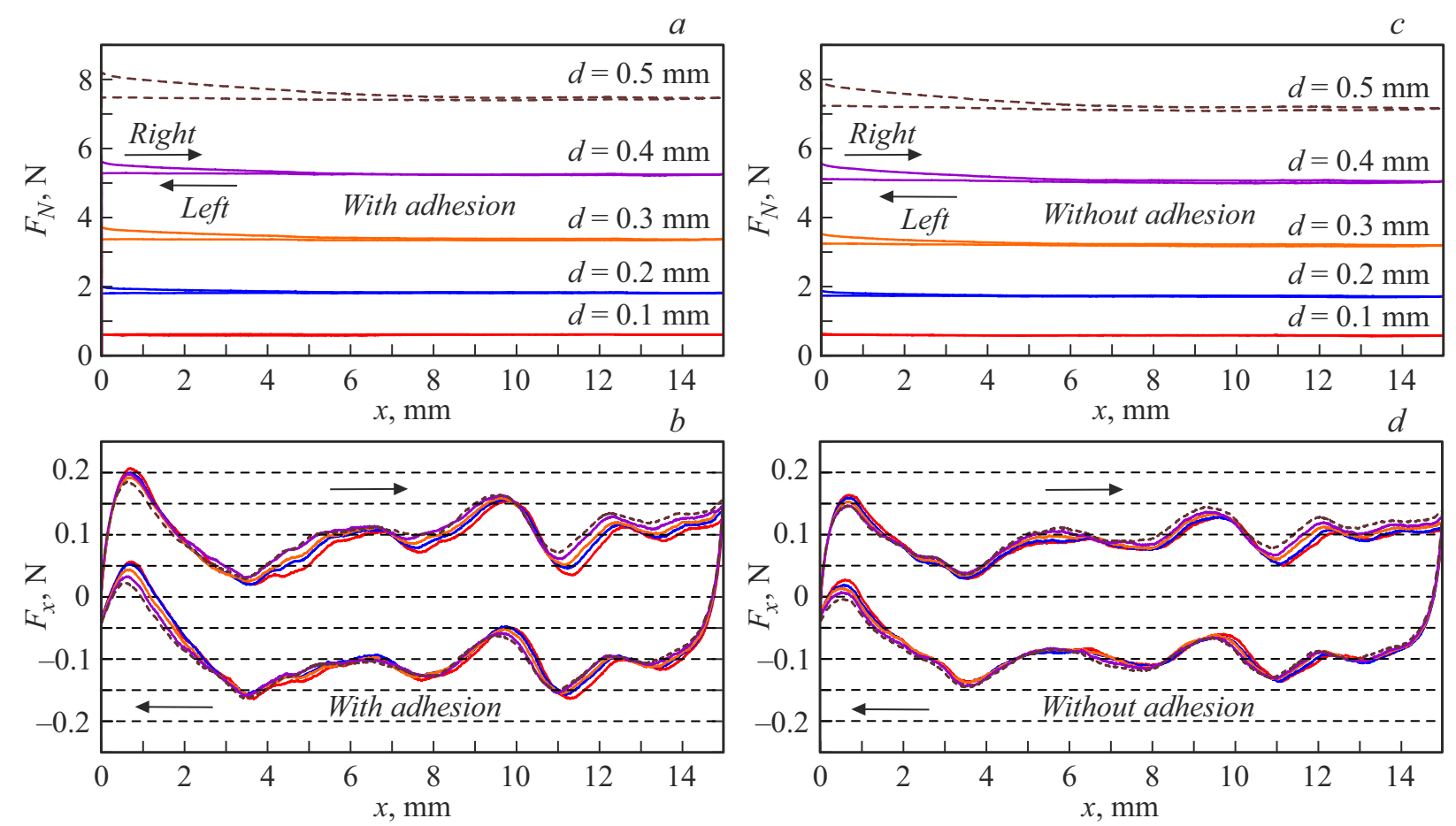

Рис. 6. Зависимости нормальной $F_{N}$ и тангенциальной $F_{x}$ контактных сил от тангенциального смещения индентора $x$ при различных значениях глубины индентирования $d: a$ и $b$ соответствуют случаю наличия адгезии между контактирующими телами; $c$ и $d$ отвечают безадгезионному случаю.
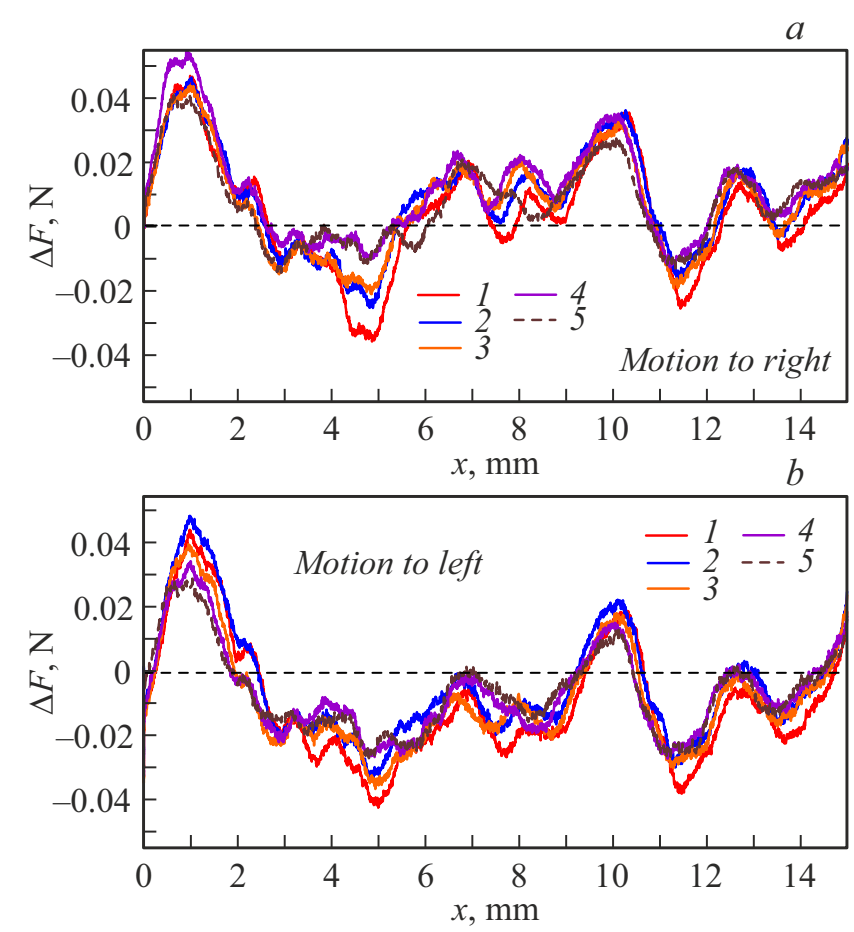

Рис. 7. Зависимости разницы $\Delta F$ между тангенциальными компонентами сил $F_{x}(x)$ для адгезионного (рис. $\left.6, b\right)$ и безадгезионного (рис. $6, d$ ) случаев при движении индентора вправо (верхняя панель) и влево (нижняя панель). показывал понижение адгезионного вклада в измеряемую тангенциальную силу $F_{x}$.

На рис. 7 приведены зависимости адгезионных вкладов в тангенциальную силу, представляющие разницу между зависимостями $F_{x}(x)$, показанными на рис. 6, для всех пяти значений глубин индентирования $d$. Из рисунка видно, что адгезионный вклад не зависит от величины глубины индентирования. Рассчитанные значения $\Delta F$, показанные на рис. 7 , существенно меньше аналогичных величин, представленных на рис. 4. Как указано выше, такая особенность наблюдается из-за понижения удельной работы адгезии, происходящей за счет загрязнения поверхностей резины и индентора.

\section{4. Тангенциальный сдвиг - скольжение}

В предыдущих разделах работы рассматривается система, в которой контактирующее с поверхностью эластомера колесо вращается вокруг своей оси за счет наличия шарикового подшипника качения. При этом на вид измеряемых зависимостей тангенциальной силы $F_{x}$ от координаты смещения колеса $x$ существенно влияют процессы, проходящие внутри подшипника. В этом разделе мы описываем результаты экспериментов, в которых колесо было жестко зафиксировано относительно своей оси и не могло вращаться. Приведенные на рис. 8 зависимости соответствуют скольжению колеса 

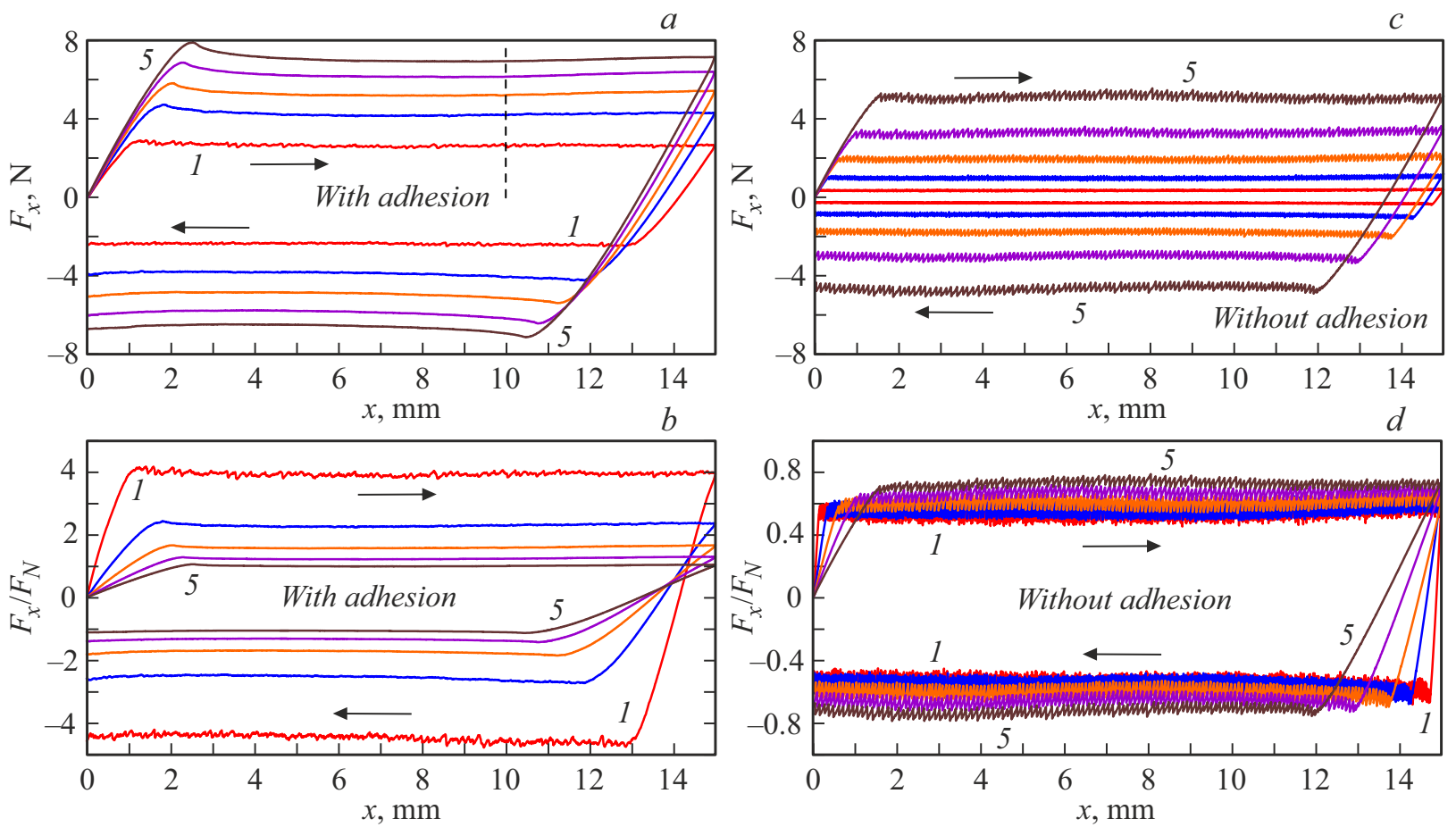

Рис. 8. Зависимости тангенциальной $F_{x}$ контактной силы и отношения тангенциальной силы к нормальной $F_{x} / F_{N}$ от тангенциального смещения индентора $x$ при различных значениях глубины индентирования $d$. Панели $a$ и $b$ соответствуют случаю наличия адгезии между контактирующими телами; $c$ и $d$ отвечают безадгезионному случаю. Различные кривые соответствуют значениям глубины индентирования $d=0.1,0.2,0.3,0.4$ и $0.5 \mathrm{~mm}$. В прилагаемом к статье видео файле (пункт 3 Приложения) показан ход экспериментов, где продемонстрированы зависимости от времени нормальной и тангенциальной сил, а также зависимость тангенциальной силы от координаты, дополненные фотографиями контактной области. Видео демонстрирует как адгезионный $(a, b)$, так и безадгезионный $(c, d)$ случаи для глубины индентирования $d=0.2 \mathrm{~mm}$. Видео доступно в онлайн-версии статьи.

(цилиндрического индентора) без вращения. В остальном эксперимент был проведен по той же методике, что и описанные выше. Серия похожих экспериментов, в которых изучалось трение скольжения между сферическим индентором и эластомером, была описана в предыдущей работе [6].

Рис. $8, a$ и $b$ соответствуют ситуации, когда индентор вдавливался в резиновый образец, тщательно очищенный от пыли из мела, используемой в предыдущих экспериментах, поэтому в данном случае присутствует адгезионное взаимодействие между поверхностями контактирующих тел. На рисунках показаны 5 зависимостей, для глубин индентирования $d$ от 0.1 до $0.5 \mathrm{~mm}$ с шагом $0.1 \mathrm{~mm}$. Кривые под номером 1 соответствуют значению $d=0.1 \mathrm{~mm}$, кривые 5 - глубине $d=0.5 \mathrm{~mm}$, остальные кривые расположены последовательно между ними. Как видно из рис. $8, b$, в рассматриваемом случае возникающая в контакте сила трения $F_{x}$ не может быть охарактеризована коэффициентом трения $\mu=\left|F_{x} / F_{N}\right|$, поскольку определенный таким образом коэффициент трения становится зависимым от значения глубины индентирования $d$. Определим для всех случаев действующие в контакте тангенциальные напряжения $\tau$. Расчет напряжений приведем для моментов времени, со- ответствующих сдвигу индентора вправо на расстояние $x=10 \mathrm{~mm}$ (на рис. 8, $a$ это положение индентора показано вертикальной штриховой линией). Проведенные эксперименты показали, что при тангенциальном движении с фиксированным значением глубины индентирования $d$ в стационарном режиме площадь контакта $A$ изменяется незначительно, испытывая изменения возле некоторого среднего значения. Тангенциальная сила $F_{x}$ в стационарном режиме также близка к постоянному значению, что видно на рис. $8, a$. Поэтому средние значения тангенциальных напряжений $\tau=F_{x} / A$ для каждой заданной глубины индентирования $d$ в стационарном режиме трения могут быть использованы в качестве характеристики режима трения. В адгезионном случае (рис. 8, $a$ и $b$ ) для глубины индентирования $d$ от 0.1 до $0.5 \mathrm{~mm}$ наблюдаемые площади контакта составили $\langle A\rangle \approx 47,74,91,109$ и $120 \mathrm{~mm}^{2}$. Стационарные значения тангенциальных сил, соответствующие этим же глубинам индентирования и смещению индентора $x$, $F_{x} \approx 2.65,4.2,5.23,6.18$ и $6.97 \mathrm{~N}$. В результате получим напряжения $\tau=F_{x} / A \approx 56.4,56.8,57.5,56.7$ и $58.1 \mathrm{kPa}$. Полученные значения свидетельствуют о том, что вне зависимости от глубины индентирования $d$ тангенциальные напряжения остаются практическими постоянными 
и являются характеристикой режима трения. Поэтому мы имеем дело с режимом, в котором сила трения задается формулой $F_{x} \approx \tau A$, т. е. пропорциональна площади контакта. При этом коэффициент трения $\mu$, как это указано выше, теряет смысл. Аналогичные результаты были получены в работе [6], где изучалось скольжение сферического индентора.

На рис. 8,c показаны результаты эксперимента, в котором поверхность резины была покрыта тонким слоем пыли из мела, с целью „выключения“ адгезионного взаимодействия. Из сравнения рис. 8, $a$ и $c$ следует, что адгезионный и безадгезионный случаи показывают качественно различное поведение. При наличии адгезионного взаимодействия (рис. 8, $a$ ) в установившемся режиме сила трения принимает постоянное значение. В безадгезионном случае (рис. 8,c) для всех глубин индентирования $d$ наблюдался периодический прерывистый (stick-slip) режим трения. Сила трения $F_{x}$ в этом случае ниже, что говорит о том, что адгезия приводит к дополнительной диссипации механической энергии. На рис. 8, $d$ приведены рассчитанные значения отношений между тангенциальной и нормальной компонентами контактных сил. Полученные значения $\mu=\left|F_{x} / F_{N}\right|$ для всех глубин индентирования $d$ находятся в диапазоне от 0.45 до 0.78 , который гораздо меньше диапазона, соответствующего адгезионному случаю (рис. 8,b), поэтому в безадгезионном случае в качестве характеристики контакта целесообразно использование коэффициента трения. Однако проведенные эксперименты все же показали, что в безадгезионном случае коэффициент трения $\mu$ имеет тенденцию к увеличению с ростом глубины индентирования $d$ (рис. $8, d$ ). Это может быть связано с дополнительной диссипацией механической энергии за счет вязкости, поскольку наблюдаемый stick-slipрежим связан с прохождением в контакте упругих волн. Скорость распространения волн на несколько порядков превышает скорость движения индентора $v=5 \mu \mathrm{m} / \mathrm{s}$, поэтому в момент прохождения волны вклад вязко-упругих эффектов в силу трения может оказаться существенным.

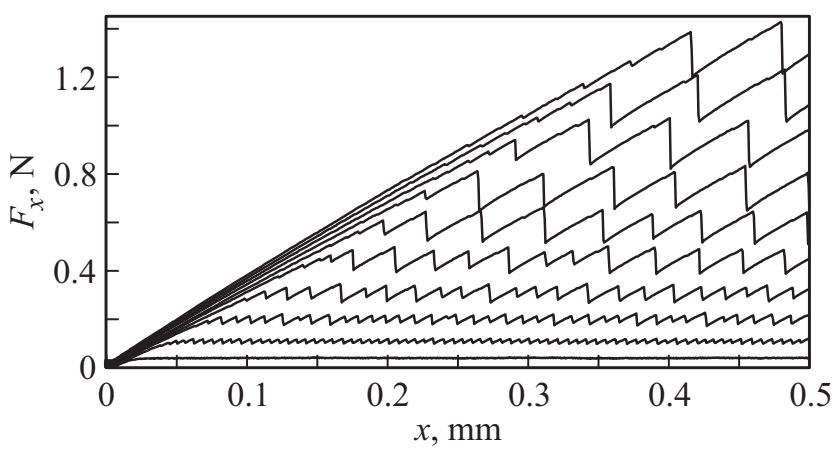

Рис. 9. Зависимости тангенциальной $F_{x}$ контактной силы от тангенциального смещения индентора $x$ для безадгезионного случая при различных значениях глубины индентирования $d$. Кривые, расположенные снизу вверх, отвечают значениям $d$ от 0.025 до $0.25 \mathrm{~mm}$ с шагом $0.025 \mathrm{~mm}$ (всего 10 кривых).
Анализ вклада вязкости в силу трения в stick-slipрежиме требует более тщательного изучения, которое мы планируем провести в последующих работах.

С целью более подробного анализа особенностей stick-slip-режима был проведен дополнительный эксперимент, результаты которого показаны на рис. 9. В этом эксперименте после окончания фазы нормального индентирования индентор сдвигался в тангенциальном направлении на величину $0.5 \mathrm{~mm}$. На рисунке показаны зависимости силы трения $F_{x}(x)$ для десяти значений глубины индентирования $d$. Кривые, расположенные снизу вверх, отвечают увеличению $d$. Было обнаружено, что с увеличением глубины индентирования увеличивается амплитуда stick-slip-переходов, а также уменьшается их частота. С увеличением $d$ возрастает контактное давление, а возрастание амплитуды и уменьшение частоты stick-slipпереходов с увеличением давления многократно наблюдалось экспериментально для различных систем [22,23]. Связано это с тем, что с ростом контактного давления увеличивается критическое значение статической силы трения, которая должна быть преодолена для осуществления фазы проскальзывания.

\section{5. Оценка значения коэффициента трения скольжения при наличии адгезии}

\section{1. Теоретическая оценка}

На рис. $8, a$ и $b$ показаны зависимости силы трения $F_{x}$ и ее отношения к нормальной силе $F_{x} / F_{N}$ от величины тангенциального смещения индентора $x$ при наличии адгезии между индентором и эластомером. Известно, что тангенциальный сдвиг в адгезионном контакте уменьшает площадь контакта [14,24]. С другой стороны, в процессе нормального индентирования в адгезионном контакте площадь контакта больше, чем в контакте без адгезии, хотя используемые нами материалы в фазе вдавливания показывают незначительный рост площади контакта за счет адгезии (рис. 2 и пояснение к нему). Проведем теоретическую оценку площади контакта без учета указанных эффектов. Оценку будем производить для более простого случая контакта между упругим полупространством с модулем упругости $E$ и коэффициентом Пуассона $v$ и жестким параболическим индентором с радиусом $R$, поскольку для этого случая известны все необходимые аналитические решения (контакт Герца [25]). При вдавливании индентора площадь контакта определится как

$$
A=\pi R d
$$

что в случае реализации по всему контакту постоянных тангенциальных напряжений $\tau_{0}$ дает силу трения

$$
F_{x}=A \tau_{0}=\pi R d \tau_{0} .
$$


Нормальная сила задается равенством

$$
F_{N}=\frac{4 E R^{1 / 2}}{3\left(1-v^{2}\right)} d^{3 / 2}
$$

Коэффициент трения в рассматриваемой ситуации определится как $\mu=F_{x} / F_{N}$ :

$$
\mu=\frac{3 \pi R^{1 / 2}\left(1-v^{2}\right) \tau_{0}}{4 E d^{1 / 2}}
$$

Согласно (4), в случае, когда вне зависимости от глубины индентирования $d$ в контакте реализуются постоянные тангенциальные напряжения $\tau_{0}$, формально определенный коэффициент трения $\mu=F_{x} / F_{N}$ уменьшается с увеличением глубины индентирования. Именно это мы и наблюдали в эксперименте (рис. $8, b)$. Отметим, что формула (4) приводит к бесконечному значению коэффициента трения при нулевой глубине индентирования. Этот предельный случай является логичным, поскольку адгезионный контакт и тангенциальная сдвиговая сила будут иметь место даже при отсутствии нормальной нагрузки. Более того, если учесть адгезионное взаимодействие, то нулевой глубине индентирования будет отвечать отрицательная нормальная сила при положительной тангенциальной составляющей (противоположной к направлению движения). А такая ситуация приводит к значениям $\mu<0$, что также наблюдалось в эксперименте [6].

\section{2. Сравнение с экспериментом}

На рис. 8, $b$ приведены экспериментально найденные зависимости коэффициента трения $\mu$ для четыpex значений глубины индентирования $d$. Имеющиеся значения позволяют провести сравнение экспериментальных результатов с теоретическим предсказанием. Полученное выше соотношение (4) описывает контакт полупространства и параболического индентора и может быть использовано только для качественного анализа, поскольку в предлагаемой работе мы описываем результаты экспериментов по контакту цилиндрического индентора с листом резины ограниченной толщины. Из эксперимента, результаты которого показаны на рис. $8, b$, следует, что при тангенциальном движении в контакте вне зависимости от глубины индентирования $d$ устанавливается постоянное значение тангенциальных напряжений $\tau_{0}$. Сдвиговые напряжения в этом случае являются характеристикой контакта, поскольку задают силу трения $F_{x}=A \tau_{0}$, где $A-$ площадь контакта. Коэффициент трения определится как

$$
\mu=\frac{F_{x}}{F_{N}}=\frac{A \tau_{0}}{F_{N}} .
$$

Сдвиговые напряжения, определенные в эксперименте, составляют $\tau_{0} \approx 57.1 \mathrm{kPa}$ (величина усреднена по пяти значениям, приведенным выше по тексту для различных глубин индентирования, см. описание рис. 8, $a, b)$.

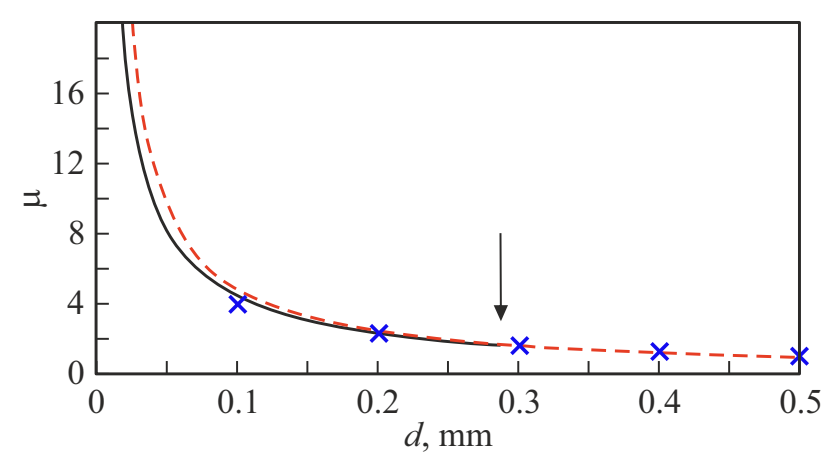

Рис. 10. Зависимости коэффициента трения $\mu=F_{x} / F_{N}$ от глубины индентирования $d$ для агдезионного контакта. Сплошная кривая показывает расчет с использованием экспериментальных результатов для фазы индентирования в нормальном направлении, приведенных на рис. 2. Штриховая кривая соответствует результатам компьютерного моделирования в фазе индентирования, результаты которого также показаны на рис. 2. Символы - результаты экспериментов, проиллюстрированных на рис. 8.

В качестве площади контакта $A$ и нормальной силы $F_{N}$ будем использовать значения, полученные в эксперименте по нормальному индентированию, приведенные на рис. 2, для фазы индентирования. В эксперименте нами неоднократно наблюдалось, что после завершения фазы нормального индентирования при дальнейшем тангенциальном сдвиге нормальная сила и площадь контакта изменяются незначительно [6] (если глубина индентирования не близка к нулевой и не отрицательна, когда контакт существует в основном или только за счет адгезии). На рис. 10 сплошной линией показана зависимость коэффициента трения $\mu$ от глубины индентирования $d$, определенная по формуле (5), где использовалось экспериментальное значение $\tau_{0} \approx 57.1 \mathrm{kPa}$, а в качестве зависимостей площади контакта $A(d)$ и нормальной силы $F_{N}(d)$ - экспериментальные зависимости для фазы индентирования в нормальном контакте, приведенные на рис. 2. Сплошная кривая на рисунке продолжается до значения глубины $d$, показанной стрелкой, поскольку это значение соответствует максимальной глубине индентирования в эксперименте (рис. 2). Штриховая кривая продолжается дальше, она была получена при использовании в качестве $A(d)$ и $F_{N}(d)$ зависимостей, соответствующих моделированию фазы индентирования. Частично результаты моделирования показаны символами на рис. 2. Для построения штриховой зависимости, приведенной на рис. 10, было проведено дополнительное моделирование процесса индентирования до глубины $d=0.5 \mathrm{~mm}$ при параметрах рис. 2. И наконец, символами на рис. 10 показаны экспериментальные значения коэффициента трения в стационарном режиме, соответствующие данным, показанным на рис. $8, b$, для величины сдвига индентора $x=10 \mathrm{~mm}$ (на рис. $8, a$ этот момент показан вертикальной штриховой линией). Из рис. 10 следует, что коэффициент трения, определенный 
непосредственно в эксперименте по тангенциальному движению индентора, с удовлетворительной точностью совпадает со значением, которое предсказывает эксперимент по нормальному индентированию, а также с результатами компьютерного моделирования. При этом зависимости $\mu(d)$ демонстрируют уменьшение коэффициента трения с увеличением глубины индентирования от бесконечности до нуля.

Интересным является то, что при значениях $d$, близких к нулевым, нормальная сила становится отрицательной, что формально приводит к отрицательным значениям коэффициента трения. Общее поведение, свойственное таким экспериментам, показывает, что при малых глубинах индентирования тангенциальный сдвиг приводит к существенному уменьшению величины площади контакта [6], что не позволяет определить коэффициент трения, используя данные моделирования нормального контакта. Например, в случае отрицательной глубины индентирования (положительное расстояние между индентором и эластомером) тангенциальный сдвиг полностью разрушает существующий за счет адгезии контакт [6], что тривиально приводит к исчезновению трения. Хотя при этом результаты по нормальному индентированию будут показывать наличие контакта и нормальной силы. Исследование зависимости формально определенного коэффициента трения от глубины индентирования при близких к нулю значениях $d$ представляет отдельную задачу, и мы планируем провести такое исследование в будущих работах.

Из проведенного в этом разделе исследования можно сделать практический вывод. Предположим, что мы имеем дело с системой, в которой нужно уменьшить коэффициент трения в сухом контакте без использования смазочных материалов. При этом для малых нормальных контактных нагрузок (малые глубины индентирования) выгодней создавать безадгезионный контакт. Однако если нагрузка (и соответственно глубина индентирования) превышают некоторую критическую величину, более целесообразно в контактной паре использовать материалы с наличием адгезионного взаимодействия, поскольку коэффициент трения в таких системах падает с увеличением $d$.

\section{Выводы}

В работе рассмотрены режимы трения качения и скольжения с наличием и отсутствием адгезии между контактирующими телами. Показано, что при наличии адгезионного взаимодействия при тангенциальном движении в режиме скольжения устанавливается постоянное значение силы трения, а в контакте реализуются постоянные тангенциальные напряжения, поэтому его нельзя охарактеризовать коэффициентом трения. В безадгезионном случае, когда поверхность резины была покрыта тонким слоем пыли из мела, реализуется stick-slipрежим. Сила трения при этом ниже, чем сила трения при наличии адгезии. Случай отсутствия адгезионного взаимодействия может быть охарактеризован коэффициентом трения, который, однако, немного возрастает с увеличением глубины индентирования. Такое увеличение мы связываем с дополнительной диссипацией механической энергии при прохождении в контакте в фазе „slip“ упругих волн со скоростями, значительно превышающими значения, при которых контакт можно считать квазистатическим. Таким образом, наличие адгезионного взаимодействия между контактирующими телами приводит к подавлению прерывистого режима трения, который часто является нежелательным, поскольку приводит к преждевременному износу оборудования. Но при этом адгезия увеличивает диссипацию механической энергии, что выражается в увеличении силы трения.

В случае трения качения наличие адгезии также приводит к увеличению значения силы трения, но не приводит к качественному изменению поведения изучаемой системы. Мы полагаем, что для наблюдения качественных отличий необходимо гораздо более сильное адгезионное взаимодействие, имеющее место между очищенными от загрязнений и оксидов поверхностями. Мы планируем изучить особенности трения качения с повышенной адгезией в последующих работах. Многократное увеличение адгезии может быть достигнуто не только очисткой индентора, но и специальной обработкой его поверхности — например, кратковременное травление в водном растворе хлорного железа. Отметим, что многочисленные эксперименты показали, что такие поверхности при контактном взаимодействии быстро утрачивают свои первоначальные адгезионные свойства, поэтому контакт свежеприготовленных поверхностей имеет скорее фундаментальное, чем практическое значение.

\section{Приложение}

В настоящей работе содержатся экспериментальные результаты, полученные с помощью установки, представленной на рис. 1. Во всех проведенных экспериментах, помимо измерения контактных сил, каждую секунду сохранялись фотографии контактной области. Для некоторых экспериментов, описанных в настоящей работе, были созданы видеофайлы, которые показывают эволюцию контактных сил и формы контакта. Данные видеофайлы являются ценным дополнением, поскольку позволяют проследить эксперимент в динамике. Файлы размещены по постоянным ссылкам с использованием платформы ResearchGate, которая позволяет присваивать классификаторы DOI цифровым объектам. В этом Приложении мы приводим ссылки на указанные видеофайлы с их описанием.

1) https://doi.org/10.13140/RG.2.2.20690.07363 Видео, демонстрирующее результаты эксперимента по индентированию в нормальном направлении жесткого цилиндрического индентора, имеющего радиус $R=80 \mathrm{~mm}$ и 
ширину $H=11 \mathrm{~mm}$, в упругий лист прозрачной резины TARNAC CRG N3005 с толщиной $h=5 \mathrm{~mm}$. Левая панель показывает конфигурацию зоны контакта, на ней же в секундах приведено реальное время $t$, прошедшее от начала индентирования. Также на видео показаны зависимости нормальной силы $F$ (средняя панель) и площади контакта $A$ (правая панель) от глубины индентирования $d$. Видео демонстрирует результаты трех подряд проведенных циклов индентирования. Видеофайл соответствует рис. 2 в статье.

2) https://doi.org/10.13140/RG.2.2.34111.84644

Видео, демонстрирующее результаты эксперимента по исследованию трения качения жесткого цилиндрического индентора, имеющего радиус $R=80 \mathrm{~mm}$ и ширину $H=11 \mathrm{~mm}$, индентированного в упругий лист прозрачной резины TARNAC CRG N3005 с толщиной $h=5 \mathrm{~mm}$ на глубину $d=0.2 \mathrm{~mm}$. Верхняя панель показывает конфигурацию зоны контакта, на ней также приведены значения глубины индентирования $d$ и тангенциального сдвига $x$. В видео показаны зависимости нормальной силы $F_{N}$ (Normal force) и тангенциальной силы $F_{x}$ (Shear force) от времени $t$, выраженного в минутах, а также зависимость тангенциальной силы $F_{x}$ от смещения индентора $x$. Подряд показаны два эксперимента - в первом реализуется непосредственный контакт поверхности индентора с резиной („адгезионный“ случай), а во втором эксперименте поверхность резины покрыта тонким слоем пыли из мела („безадгезионный“ случай). Видеофайл соответствует рис. 3 в статье.

3) https://doi.org/10.13140/RG.2.2.25723.23840

Описание видео файла совпадает с описанием пункта 2, с тем отличием, что в этом эксперименте колесо (индентор) было жестко закреплено на оси и не могло вращаться. Поэтому представленный на видео эксперимент соответствует трению скольжения.

\section{Благодарности}

Настоящая работа выполнена при частичной финансовой поддержке Deutsche Forschungsgemeinschaft (проект РО 810/55-3), а также государственного задания ИФРПМ СО РАН.

\section{Конфликт интересов}

Авторы заявляют, что у них нет конфликта интересов.

\section{Список литературы}

[1] Handbook of adhesion technology, ed. by L.F.M. da Silva, A. Öchsner, R.D. Adams, 2-nd ed. (Springer, Berlin, 2018), DOI: $10.1007 / 978-3-319-55411-2$

[2] A.M. Krachler, K. Orth. Virulence, 4 (4), 284 (2014). DOI: 10.4161/viru.24606

[3] C. Berne, C.K. Ellison, A. Ducret, Y.V. Brun. Nat. Rev. Microbiol., 16, 616 (2018). DOI: 10.1038/s41579-018-0057-5
[4] Adhesion and Friction in Biological Systems, ed. by S. Gorb (Springer, Berlin, 2012)

[5] K. Autumn, N. Gravish. Phil. Trans. R. Soc. A, 366 (1870), 1575 (2008). DOI: 10.1098/rsta.2007.2173

[6] V.L. Popov, Q. Li, I.A. Lyashenko, R. Pohrt. Friction, 9(6), 1688 (2021). DOI: 10.1007/s40544-020-0482-0

[7] K.L. Johnson, K. Kendall, A.D. Roberts. Proc. Royal Soc. Lond. A, 324 (1558), 301 (1971). DOI: $10.1098 /$ rspa.1971.0141

[8] E. Popova, V.L. Popov. Phys. Mesomech., 21 (1), 1 (2018). DOI: $10.1134 / \mathrm{S} 1029959918010010$

[9] B.V. Derjaguin, V.M. Muller, Y.P. Toporov. J. Colloid Interf. Sci., 53 (2), 314 (1975). DOI: 10.1016/0021-9797(75)90018-1

[10] D. Maugis. J. Colloid Interf. Sci., 150(1), 243 (1992). DOI: 10.1016/0021-9797(92)90285-T

[11] W. Deng, H. Kesari. Sci. Rep. 9, 1639 (2019). DOI: $10.1038 / \mathrm{s} 41598-018-38212-\mathrm{z}$

[12] S. Dalvi, A. Gujrati, S.R. Khanal, L. Pastewka, A. Dhinojwala, T.D.B. Jacobs. Proc. Natl. Acad. Sci. USA, 116 (51), 25484 (2019). DOI: 10.1073/pnas.1913126116

[13] S. You, M.P. Wan. Langmuir, 30 (23), 6808 (2014). DOI: $10.1021 / 1 a 500360 f$

[14] M. Ciavarella, A. Papangelo. Tribol. Lett., 68, 81 (2020). DOI: $10.1007 / \mathrm{s} 11249-020-01318-5$

[15] H. Radhakrishnan, S. Akarapu. Sci. Rep., 10, 5402 (2020). DOI: $10.1038 / \mathrm{s} 41598-020-61187-9$

[16] G. Violano, L. Afferrante. Tribol. Lett., 67, 119 (2019). DOI: $10.1007 / \mathrm{s} 11249-019-1232-1$

[17] J.A. Greenwood. Tribol. Lett., 65, 159 (2017). DOI: $10.1007 / \mathrm{s} 11249-017-0938-1$

[18] Я.А. Ляшенко, В.Л. Попов. Письма в ЖТФ, 46 (21), 44 (2020). DOI: 10.21883/PJTF.2020.21.50197.18431 [I.A. Lyashenko, V.L. Popov. Tech. Phys. Lett., 46 (11), 1092 (2020). DOI: 10.1134/S1063785020110097]

[19] I.A. Lyashenko, R. Pohrt. Front. Mech. Eng., 6, 49 (2020). DOI: $10.3389 /$ fmech.2020.00049

[20] Я.А. Ляшенко, В.Л. Попов. ЖТФ, 91 (4), 689 (2021). DOI: 10.21883/JTF.2021.04.50634.197-20 [I.A. Lyashenko, V.L. Popov. Tech. Phys., 66 (4), 672 (2021). DOI: $10.1134 / \mathrm{S} 1063784221040113]$

[21] Q. Li, R. Pohrt, I.A. Lyashenko, V.L. Popov. Proc. Inst. Mech. Eng. J., 234 (1), 73 (2020). DOI: 10.1177/1350650119854250

[22] M.Y. Thomas, T.M. Mitchell, H.S. Bhat (editors). Fault Zone Dynamic Processes: Evolution of Fault Properties During Seismic Rupture (Wiley, USA, 2017), DOI: 10.1002/9781119156895, Ch. 6, D.A. Lockner, B.D. Kilgore, N.M. Beeler, D.E. Moore. The transition from Frictional Sliding to Shear Melting in Laboratory Stick-Slip Experiments P. 105-131 (2017). DOI: $10.1002 / 9781119156895 . c h 6$

[23] C. Dong, C. Yuan, A. Xu, X. Bai, Y. Tian. Langmuir, 35 (7), 2878 (2019). DOI: 10.1021/acs.langmuir.8b04068

[24] J.F. Waters, P.R. Guduru. Proc. Royal Soc. A, 466, 1303 (2010). DOI: 10.1098/rspa.2009.0461

[25] H.J. Hertz. J. für die reine Angew. Math., 92, 156 (1882). DOI: $10.1515 /$ crll.1882.92.156 